\title{
Batch Arrival, Single Server with Two Stage Heterogeneous Service, Bernoulli Schedule Server Vacation Feed Back, Random Break Down and Delayed Repair Time
}

\author{
Dr. G. Ayyappan ${ }^{1}$, S. Shyamala ${ }^{2}$ \\ ${ }^{I}$ (Mathematics Department, Pondicherry Engineering College /Pondicherry University, India) \\ ${ }^{2}$ (Mathematics Department, Arunai Engineering College /Pondicherry University, India)
}

\begin{abstract}
We present a single server with two heterogeneous service stages having different general (arbitrary) distribution, subject to random breakdowns and Bernoulli scheduled server vacation. The customers arrive in batches and the server provides service one by one. The second stage service must be provided after completing the first stage service by the server. On completion of the first phase of the service with FCFS schedule, the second phase starts. With probability $p$ the customer feedback to the tail of original queue for repeating the service until the service be successful. With probability $1-p=q$ the customer departs the system if service be successful. Upon the completion of the second stage service, the server will go for vacation with probability $\theta$ or staying back in the system for providing the service to the next customer with probability $1-\theta$, if any. The vacation time follows general (arbitrary) distribution. The system may breakdown at random time and the breakdowns occur according to Poisson stream. Once the server breakdown, it could not be repaired immediately, so that there is a waiting time, called 'delay time' before the server getting repaired. Both the delay time and repair time follow exponential distribution. We obtain the time dependent probability generating functions in terms of their Laplace transforms and the corresponding steady state results explicitly. Also we derive the average number of customers in the queue and the average waiting time in closed form.
\end{abstract}

Keywords: $M^{[X]} / G / 1$ Queue, Heterogeneous Service, Bernoulli Vacation, Probability Generating Function, Delay Time, Mean Queue Length.

\section{Introduction}

Due to the improvement and advancement of science and technology, performance in modeling is one of the vital parts that affect the design, configuration and implementation of any real time system. Queueing modeling is being used tremendously and effectively in congestion problems encountered in day to day life as well as industrial scenario including computer systems, web services and communication networks, waiting lines at airports, railway stations, banks etc. The study of queueing systems with feed back have been concentrated by many authors. Choudhury and Paul [8] inspected the M/G/1 system with two phase of heterogeneous service and Bernoulli feedback. In this system a customer may get an unsuccessful service, then it retries to take the service until a successful service. With probability $p(0<p<1)$ the customer feedback instantaneously to the tail of the queue or depart from the system with probability $q=1-p$. In most of the research study of queueing models, the server is assumed to be reliable such that the server works for ever, but this is not the case in most of the real scenarios that the servers are reliable such that the servers may meet breakdowns. Also there are numerous papers on queueing models with vacations and breakdowns. Baba[3] studied about batch arrival single server with vacation. A comprehensive survey can be found in Doshi[11]. Igaki[12] analyzed two servers with exponential service with $\mathrm{N}$ policy and general vacation. Keilson et al.[15] studied the dynamics of non-Markovian vacation. Maraghi et al.[22] have obtained steady state solution of batch arrival queueing system with random breakdowns and Bernoulli schedule server vacations having general vacation time.

Many researchers have paid their attention and efforts in queueing theory by considering various aspects like two phases queue system with random break downs, Bernoulli vacation etc. Anabosi et al.[1] studied a single server queue with two types of service, Bernoulli schedule server vacations and a single vacation policy. Artalejo et al.[2] discussed about the steady state analysis of an $\mathrm{M} / \mathrm{G} / 1$ queue with repeated attempts and two-phase service. BadamchiZadeh[4] studied two phases queue system with Bernoulli feedback and Bernoulli schedule server vacation. Choudhury[7] analyzed about two phases batch arrival queueing system with a vacation time under Bernoulli schedule. Madan[20,21] discussed a single server with two types of service and deterministic server vacations as well as Bernoulli vacation. Whenever the server encounters a break down it could not be able to serve unless it should be repaired. Therefore the server should undergo a repair process, but sometimes the repair process will not be started immediately due to the non availability of the 
repairing equipment or repairmen. Such situations can also be modeled as queueing model and which has been studied by many authors. Burke[5] studied delays in single-server queues with batch input. Madan[19] studied queueing system with random failures and delayed repairs. Gautam choudhury et al.[10] discussed a batch arrival, single server queue with two phases of service subject to the server breakdown and delay time. Rehab et al.[24] have obtained the steady state solution of an $M^{[X]} / G / 1$ queue with Bernoulli schedule, general vacation times, random breakdowns, general delay times and general repair times.

Most recently the study of transient behavior in queueing systems have been growing extensively due their potential applications in which a practitioner needs to know how the system will function up to a time horizon. Takagi [28] analyzed time-dependent analysis of $\mathrm{M} / \mathrm{G} / 1$ vacation models with exhaustive service. Thangaraj[30] have obtained transient solution of two phase heterogeneous services with compulsory vacation and random break downs. In this paper we consider a queueing system wherein the customers arrive in batches and the server provides service one by one in FCFS basis. Each arriving batch has to undergo two stages of service provided by a single server and the service time for two stages is assumed to follow general distribution. As soon as the second stage of a customer's service is completed, the server may go for a vacation with probability $\theta$ or continue staying in the system to provide service to a next customer, if any, with probability $1-\theta$.

On account of, the system may subject to breakdowns, the breakdowns occur according to Poisson process. Once the system breakdown, the repair process will not be started immediately so that the system has to wait before it could be repaired, such a waiting time is known as 'delay time' which follows exponential distribution. the repair time follows exponential distribution. After the repair process complete, the server resumes its work immediately. Also whenever the system meet a break down, the customer whose service is interrupted goes back to the head of the queue. The rest of the paper is organized as follows. The mathematical description of our model is in Section 2 and equations governing the model are given in Section 3. The time dependent solution have been obtained in Section 4, the corresponding steady state results have been derived explicitly in Section 5 and the concluding remarks is in 6.

\section{Mathematical Description of the Model}

We assume the following to describe the queueing model of our study.

- Customers arrive at the system in batches of variable size in a compound Poisson process. Let $\lambda c_{i} \Delta t$ $(\mathrm{i}=1,2,3, \ldots$.$) be the first order probability that a batch of \mathrm{i}$ customers arrives at the system during a short interval of time $(t, t+\Delta t)$, where $0 \leq c_{i} \leq 1$ and $\sum_{i=1}^{\infty} c_{i}=1$ and $\lambda>0$ is the mean arrival rate of batches. The customers are served one-by-one on a "first come-first served" basis.

- Each customer undergoes two stages of heterogeneous service provided by a single server on a first come first served basis. The service time of the two stages follow different general (arbitrary) distributions with distribution function $B_{j}(v)$ and the density function $b_{j}(v), \mathrm{j}=1,2$.

- Let $\mu_{i}(x) \mathrm{dx}$ be the conditional probability of completion of the $i^{\text {th }}$ stage of service during the interval (x, $\mathrm{x}+\mathrm{dx}]$ given that elapsed service time is $\mathrm{x}$, so that

$$
\mu_{i}(x)=\frac{b_{i}(x)}{1-B_{i}(x)}, i=1,2 .
$$

and therefore, $\quad b_{i}(v)=\mu_{i}(v) e^{-\int_{0}^{v} \mu_{i}(x) d x}, i=1,2$.

- After completion of first phase service and second phase service if the customer is dissatisfied with the service for certain reason or if customer received unsuccessful service, the customer may immediately join the tail of the original queue as a feedback customer for receiving another regular service with probability $p(0<p<1)$. Otherwise the customer may depart forever from the system with probability $\mathrm{q}(=1-\mathrm{p})$. The service discipline for feedback and newly customers are first come first served. Also service time for a feedback customer is independent of its previous service times.

- As soon as the second stage service of a customer is completed, the server may go for a vacation of random length $\mathrm{V}$ with probability $\theta(0 \leq \theta \leq 1)$ or it may continue to serve the next customer $(1-\theta)$. 
- The vacation time also follow general (arbitrary) distribution with distribution function $\mathrm{V}(\mathrm{s})$ and the density function $\mathrm{v}(\mathrm{s})$. Let $\gamma(x) d x$ be the conditional probability of a completion of a vacation during the interval ( $\mathrm{x}, \mathrm{x}$ $+\mathrm{dx}$ ] given that the elapsed vacation time is $\mathrm{x}$, so that

$$
\gamma(x)=\frac{v(x)}{1-V(x)}
$$

and therefore, $\quad v(s)=\gamma(s) e^{-\int_{0}^{s} \gamma(x) d x}$

- On returning from vacation the server instantly starts serving the customer at the head of the queue, if any.

- The system may break down at random and breakdowns are assumed to occur according to a Poisson stream with mean breakdown rate $\alpha>0$.

- Whenever the system breakdown, its repairs do not start immediately and there is a delay time. The delay times follows exponential distribution with mean time $\frac{1}{\eta}$

- The repair time of the server is exponentially distributed with mean time $\frac{1}{\beta}$.

- Various stochastic processes involved in the system are assumed to be independent of each other.

We let,

\section{Definitions And Equations Governing The System}

(i) $P_{n}^{(1)}(x, t)=$ Probability that at time ' $\mathrm{t}$ ' the server is active providing first stage service and there are ' $\mathrm{n}$ ' $(n \geq 0)$ customers in the queue excluding the one being served and the elapsed service time for this customer is x. Consequently $P_{n}^{(1)}(t)$ denotes the probability that at time ' $\mathrm{t}$ ' there are ' $\mathrm{n}$ ' customers in the queue excluding the one customer in the first stage service irrespective of the value of $\mathrm{x}$.

(ii) $P_{n}^{(2)}(x, t)=$ Probability that at time 't' the server is active providing second stage service and there are 'n' $(n \geq 0)$ customers in the queue excluding the one being served and the elapsed service time for this customer is x. Consequently $P_{n}^{(2)}(t)$ denotes the probability that at time ' $\mathrm{t}$ ' there are ' $\mathrm{n}$ ' customers in the queue excluding the one customer in the second stage service irrespective of the value of $\mathrm{x}$.

(iii) $V_{n}(x, t)=$ probability that at time ' $\mathrm{t}$ ' the server is on vacation with elapsed vacation time $\mathrm{x}$, and there are 'n' $(n \geq 0)$ customers waiting in the queue for service. Consequently $V_{n}(t)$ denotes the probability that at time ' $t$ ' there are ' $n$ ' customers in the queue and the server is on vacation irrespective of the value of $x$.

(iv) $D_{n}(t)=$ Probability that at time $\mathrm{t}$, the server is inactive due to breakdown and the system is under waiting time before the server getting repaired while there are 'n' $(n \geq 0)$ customers in the queue.

(v) $R_{n}(t)=$ Probability that at time $\mathrm{t}$, the server is inactive due to breakdown and the system is under repair while there are 'n' $(n \geq 0)$ customers in the queue.

$(v i) Q(t)=$ probability that at time ' $t$ ' there are no customers in the system and the server is idle but available in the system.

The queueing model is then, governed by the following set of differential-difference equations:

$$
\begin{aligned}
& \frac{\partial}{\partial t} P_{n}^{(1)}(x, t)+\frac{\partial}{\partial x} P_{n}^{(1)}(x, t)+\left(\lambda+\mu_{1}(x)+\alpha\right) P_{n}^{(1)}(x, t)=\lambda \sum_{i=1}^{n-1} c_{i} P_{n-i}^{(1)}(x, t) \quad n \geq 1(5) \\
& \frac{\partial}{\partial t} P_{0}^{(1)}(x, t)+\frac{\partial}{\partial x} P_{0}^{(1)}(x, t)+\left(\lambda+\mu_{1}(x)+\alpha\right) P_{0}^{(1)}(x, t)=0 \\
& \frac{\partial}{\partial t} P_{n}^{(2)}(x, t)+\frac{\partial}{\partial x} P_{n}^{(2)}(x, t)+\left(\lambda+\mu_{2}(x)+\alpha\right) P_{n}^{(2)}(x, t)=\lambda \sum_{i=1}^{n-1} c_{i} P_{n-i}^{(2)}(x, t) \quad n \geq 1
\end{aligned}
$$




$$
\begin{aligned}
& \frac{\partial}{\partial t} P_{0}^{(2)}(x, t)+\frac{\partial}{\partial x} P_{0}^{(2)}(x, t)+\left(\lambda+\mu_{2}(x)+\alpha\right) P_{0}^{(2)}(x, t)=0 \\
& \frac{\partial}{\partial t} V_{n}(x, t)+\frac{\partial}{\partial x} V_{n}(x, t)+(\lambda+\gamma(x)) V_{n}(x, t)=\lambda \sum_{i=1}^{n-1} c_{i} V_{n-i}(x, t) \quad n \geq 1 \\
& \frac{\partial}{\partial t} V_{0}(x, t)+\frac{\partial}{\partial x} V_{0}(x, t)+(\lambda+\gamma(x)) V_{0}(x, t)=0 \\
& \frac{d}{d t} D_{n}(t)=-(\lambda+\eta) D_{n}(t)+\lambda \sum_{i=1}^{n} c_{i} D_{n-i}(t)+\alpha \int_{0}^{\infty} P_{n-1}^{(1)}(x, t) d x+\alpha \int_{0}^{\infty} P_{n-1}^{(2)}(x, t) d x \\
& \frac{d}{d t} D_{0}(t)=-(\lambda+\eta) D_{0}(t) \\
& \frac{d}{d t} R_{n}(t)=-(\lambda+\beta) R_{n}(t)+\lambda \sum_{i=1}^{n-1} c_{i} R_{n-i}(t)+\eta D_{n}(t) \\
& \frac{d}{d t} R_{0}(t)=-(\lambda+\beta) R_{0}(t)+\eta D_{0}(t) \\
& \frac{d}{d t} Q(t)=-\lambda Q(t)+\beta R_{0}(t)+\int_{0}^{\infty} V_{0}(x, t) \gamma(x) d x+(1-\theta) q \int_{0}^{\infty} P_{0}^{(2)}(x, t) \mu_{2}(x) d x
\end{aligned}
$$

Equations (5) to (15) are to be solved subject to the following boundary conditions.

$$
\begin{aligned}
& P_{0}^{(1)}(0, t)= c_{1} \lambda Q(t)+\beta R_{1}(t)+\int_{0}^{\infty} V_{1}(x, t) \gamma(x) d x \\
&+(1-\theta)\left\{p \int_{0}^{\infty} P_{0}^{(2)}(x, t) \mu_{2}(x) d x+q \int_{0}^{\infty} P_{1}^{(2)} \mu_{2}(x) d x\right\} \\
& P_{n}^{(1)}(0, t)= \beta R_{n+1}(t)+\int_{0}^{\infty} V_{n+1}(x, t) \gamma(x) d x \\
&+(1-\theta)\left\{p \int_{0}^{\infty} P_{n+1}^{(2)}(x, t) \mu_{2}(x) d x+q \int_{0}^{\infty} P_{1}^{(2)} \mu_{2}(x) d x\right\}+\lambda c_{n+1} Q(t) \\
& P_{n}^{(2)}(0, t)=\int_{0}^{\infty} P_{n}^{(1)}(x, t) \mu_{1}(x) d x \quad n \geq 0 \\
& V_{n}(0, t)=\theta \int_{0}^{\infty} P_{n}^{(2)}(x, t) \mu_{2}(x) d x \quad n \geq 0
\end{aligned}
$$

We assume that initially there is no customers in the system and the server is idle. So the initial conditions are

$$
P_{n}^{(j)}(0)=0 ; n=0,1,2 \ldots, j=1,2 ; V_{0}(0)=V_{n}(0)=0 ; Q(0)=1
$$

\section{Probability Generating Functions Of The Queue Length: The Time-Dependent Solution}

We define the probability generating function

$$
\begin{aligned}
& P_{q}^{(i)}(x, z, t)=\sum_{n=0}^{\infty} z^{n} P_{n}^{(i)}(x, t) ; P_{q}^{(i)}(z, t)=\sum_{n=0}^{\infty} z^{n} P_{n}^{(i)}(t) \\
& V_{q}(x, z, t)=\sum_{n=0}^{\infty} z^{n} V_{n}(x, t) ; V_{q}(z, t)=\sum_{n=0}^{\infty} z^{n} V_{n}(t) \\
& D_{q}(z, t)=\sum_{n=0}^{\infty} z^{n} D_{n}(t) ; R_{q}(z, t)=\sum_{n=0}^{\infty} z^{n} R_{n}(t) \\
& C(z)=\sum_{n=1}^{\infty} c_{n} z^{n}
\end{aligned}
$$

which are convergent inside the circle given by $|z| \leq 1$ and define the Laplace transform of a function $\mathrm{f}(\mathrm{t})$ as

$$
\bar{f}(s)=\int_{0}^{\infty} f(t) e^{-s t} d t
$$

Taking Laplace transforms of equations (5) to (15) 


$$
\begin{aligned}
& \frac{\partial}{\partial x} \bar{P}_{n}^{(1)}(x, s)+\left(s+\lambda+\mu_{1}(x)+\alpha\right) \bar{P}_{n}^{(1)}(x, s)=\lambda \sum_{i=1}^{n-1} c_{i} \bar{P}_{n-i}^{(1)}(x, s) \quad n \geq 1 \\
& \frac{\partial}{\partial x} \bar{P}_{0}^{(1)}(x, s)+\left(s+\lambda+\mu_{1}(x)+\alpha\right) \bar{P}_{0}^{(1)}(x, s)=0 \\
& \frac{\partial}{\partial x} \bar{P}_{n}^{(2)}(x, s)+\left(s+\lambda+\mu_{2}(x)+\alpha\right) \bar{P}_{n}^{(2)}(x, s)=\lambda \sum_{i=1}^{n-1} c_{i} \bar{P}_{n-i}^{(2)}(x, s) \quad n \geq 1 \\
& \frac{\partial}{\partial x} \bar{P}_{0}^{(2)}(x, s)+\left(s+\lambda+\mu_{2}(x)+\alpha\right) \bar{P}_{0}^{(2)}(x, s)=0 \\
& \frac{\partial}{\partial x} \bar{V}_{n}(x, s)+(s+\lambda+\gamma(x)) \bar{V}_{n}(x, s)=\lambda \sum_{i=1}^{n-1} c_{i} \bar{V}_{n-i}(x, s) \\
& \frac{\partial}{\partial x} \bar{V}_{0}(x, s)+(s+\lambda+\gamma(x)) \bar{V}_{0}(x, s)=0 \\
& (s+\lambda+\eta) \bar{D}_{n}(s)=\lambda \sum_{i=1}^{n-1} c_{i} \bar{D}_{n-i}(s)+\alpha \int_{0}^{\infty} \bar{P}_{n-1}^{(1)}(x, s) d x+\alpha \int_{0}^{\infty} \bar{P}_{n-1}^{(2)}(x, s) d x \\
& (s+\lambda+\eta) \bar{D}_{0}(s)=0 \\
& (s+\lambda+\beta) \bar{R}_{n}(s)=\lambda \sum_{i=1}^{n-1} c_{i} \bar{R}_{n-i}(s)+\eta \bar{D}_{n}(s) \\
& (s+\lambda+\beta) \bar{R}_{0}(s)=\eta \bar{D}_{0}(s) \\
& (s+\lambda) \bar{Q}(s)=1+\beta \bar{R}_{0}(s)+\int_{0}^{\infty} \bar{V}_{0}(x, s) \gamma(x) d x+(1-p) \int_{0}^{\infty} \bar{P}_{0}^{(2)}(x, s) \mu_{2}(x) d x \\
&
\end{aligned}
$$

for the boundary conditions

$$
\begin{aligned}
& \bar{P}_{0}^{(1)}(0, s)=(1-\theta)\left\{p \int_{0}^{\infty} \bar{P}_{0}^{(2)}(x, s) \mu_{2}(x) d x+q \int_{0}^{\infty} \bar{P}_{1}^{(2)}(x, s) \mu_{2}(x) d x\right\} \\
&+\int_{0}^{\infty} \overline{V_{1}}(x, s) \gamma(x) d x+\beta \bar{R}_{1}(s)+\lambda c_{1} \bar{Q}(s) \\
& \bar{P}_{n}^{(1)}(0, s)=(1-\theta)\left\{p \int_{0}^{\infty} \bar{P}_{n}^{(2)}(x, s) \mu_{2}(x) d x+q \int_{0}^{\infty} \bar{P}_{n+1}^{(2)}(x, s) \mu_{2}(x) d x\right\} \\
& \quad+\int_{0}^{\infty} \bar{V}_{n+1}(x, s) \gamma(x) d x+\beta \bar{R}_{n+1}(s)+\lambda c_{n+1} \bar{Q}(s) \\
& \bar{P}_{0}^{(2)}(0, s)=\int_{0}^{\infty} \bar{P}_{n}^{(1)}(x, s) \mu_{1}(x) d x ; n=0,1,2, \ldots \\
& \bar{V}_{0}(0, s)=p \int_{0}^{\infty} \bar{P}_{n}^{(2)}(x, s) \mu_{2}(x) d x ; n=0,1,2, \ldots
\end{aligned}
$$

Now multiplying equation (23) by $z^{n}$ and summing over $n$ from 1 to $\infty$, adding to equation (24) and using the definition of probability generating function, we obtain

$$
\frac{\partial}{\partial x} \bar{P}_{q}^{(1)}(x, z, s)+\left(s+\lambda-\lambda C(z)+\mu_{1}(x)+\alpha\right) \bar{P}_{q}^{(1)}(x, z, s)=0
$$

Performing similar operations on equations (25) to (32)

$$
\begin{aligned}
& \frac{\partial}{\partial x} \bar{P}_{q}^{(2)}(x, z, s)+\left(s+\lambda-\lambda C(z)+\mu_{2}(x)+\alpha\right) \bar{P}_{q}^{(2)}(x, z, s)=0 \\
& \frac{\partial}{\partial x} \bar{V}_{q}(x, z, s)+(s+\lambda-\lambda C(z)+\gamma(x)) \bar{V}_{q}(x, z, s)=0 \\
& (s+\lambda-\lambda C(z)+\eta) \bar{D}_{q}(z, s)=\alpha z\left[\int_{0}^{\infty} \bar{P}_{q}^{(1)}(x, z, s) d x+\int_{0}^{\infty} \bar{P}_{q}^{(2)}(x, z, s) d x\right]
\end{aligned}
$$




$$
(s+\lambda-\lambda C(z)+\beta) \bar{R}_{q}(z, s)=\eta \bar{D}_{q}(z, s)
$$

For the boundary conditions, multiply both sides of equation (34) by z, multiply both sides of equation (35) by $z^{n+1}$, summing over 1 to $\infty$, adding the two results and using the definition of probability generating function, we get,

$$
\begin{gathered}
z \bar{P}_{q}^{(1)}(0, z, s)=[1-s \bar{Q}(s)]+\lambda(C(z)-1) \bar{Q}(s)+\beta \bar{R}_{q}(z, s)+\int_{0}^{\infty} \bar{V}_{q}(x, z, s) \gamma(x) d x \\
+(p z+q)(1-\theta) \int_{0}^{\infty} \bar{P}_{q}^{(2)}(x, z, s) \mu_{2}(x) d x
\end{gathered}
$$

Performing similar operation on equations (36) and (37) we obtain

$$
\begin{aligned}
& \bar{P}_{q}^{(2)}(0, z, s)=\int_{0}^{\infty} \bar{P}_{q}^{(1)}(x, z, s) \mu_{1}(x) d x \\
& \bar{V}_{q}(0, z, s)=p \int_{0}^{\infty} \bar{P}_{q}^{(2)}(x, z, s) \mu_{2}(x) d x
\end{aligned}
$$

Integrating the equation (38) from 0 to $\mathrm{x}$ yields

$$
\bar{P}_{q}^{(1)}(x, z, s)=\bar{P}_{q}^{(1)}(0, z, s) e^{-(s+\lambda-\lambda C(z)+\alpha) x-\int_{0}^{x} \mu_{1}(t) d t}
$$

where $\bar{P}_{q}^{(1)}(0, z, s)$ is given by equation (43). Again integrating equation (46) by parts with respect to x yields

$$
\bar{P}_{q}^{(1)}(z, s)=\bar{P}_{q}^{(1)}(0, z, s)\left[\frac{1-\bar{B}_{1}(s+\lambda-\lambda C(z)+\alpha)}{(s+\lambda-\lambda C(z)+\alpha)}\right]
$$

where

$$
\bar{B}_{1}(s+\lambda-\lambda C(z)+\alpha)=\int_{0}^{\infty} e^{-(s+\lambda-\lambda C(z)+\alpha) x} d B_{1}(x)
$$

is Laplace - Stieltjes transform of the first stage service time $B_{1}(x)$. Now multiplying both sides of equation (46) by $\mu_{1}(x)$ and integrating over $\mathrm{x}$, we get

$$
\int_{0}^{\infty} \bar{P}_{q}^{(1)}(x, z, s) \mu_{1}(x) d x=\bar{P}_{q}^{(1)}(0, z, s) \bar{B}_{1}(s+\lambda-\lambda C(z)+\alpha)
$$

Similarly, on integrating equation (39) and (40) from 0 to $x$, we get

$$
\begin{aligned}
& \bar{P}_{q}^{(2)}(x, z, s)=\bar{P}_{q}^{(2)}(0, z, s) e^{-(s+\lambda-\lambda C(z)+\alpha) x-\int_{0}^{x} \mu_{2}(t) d t} \\
& \bar{V}_{q}(x, z, s)=p \bar{V}_{q}(0, z, s) e^{-\left(s+\lambda-\lambda C(z) x-\int_{0}^{x} \gamma(t) d t\right.}
\end{aligned}
$$

where $\bar{P}_{q}^{(2)}(0, z, s)$ and $\bar{V}_{q}(0, z, s)$ are given by equations (44) and (45).

Again integrating equations (50)and (51) by parts with respect to $\mathrm{x}$ yields,

where

$$
\begin{aligned}
& \bar{P}_{q}^{(2)}(z, s)=\bar{P}_{q}^{(2)}(0, z, s)\left[\frac{1-\bar{B}_{2}(s+\lambda-\lambda C(z)+\alpha)}{(s+\lambda-\lambda C(z)+\alpha)}\right] \\
& \bar{V}_{q}(z, s)=p \bar{V}_{q}(0, z, s)\left[\frac{1-\bar{V}(s+\lambda-\lambda C(z))}{(s+\lambda-\lambda C(z))}\right]
\end{aligned}
$$

$$
\bar{B}_{2}(s+\lambda-\lambda C(z)+\alpha)=\int_{0}^{\infty} e^{-(s+\lambda-\lambda C(z)+\alpha) x} d B_{2}(x)
$$

is Laplace - Stieltjes transform of the second stage service time $B_{2}(x)$, and

$$
\bar{V}(s+\lambda-\lambda C(z))=\int_{0}^{\infty} e^{-(s+\lambda-\lambda C(z) x} d V(x)
$$

is Laplace - Stieltjes transform of the vacation time $V(x)$.Now multiplying both sides of equation (50) by $\mu_{2}(x)$ and integrating over $\mathrm{x}$, we get 


$$
\int_{0}^{\infty} \bar{P}_{q}^{(2)}(x, z, s) \mu_{2}(x) d x=\bar{P}_{q}^{(2)}(0, z, s) \bar{B}_{2}(s+\lambda-\lambda C(z)+\alpha)
$$

Now using equation (49), equation (44) reduces to

$$
\bar{P}_{q}^{2}(0, z, s)=\bar{P}_{q}^{(1)}(0, z, s) \bar{B}_{1}(s+\lambda-\lambda C(z)+\alpha)
$$

Now multiplying both sides of equation (51) by $\gamma(x)$ and integrating over $\mathrm{x}$,we get

$$
\int_{0}^{\infty} \bar{V} q(x, z, s) \gamma(x) d x=p \bar{V}_{q}(0, z, s) \bar{V}(s+\lambda-\lambda C(z))
$$

Now using equations (56) and (57), equation(45) can be written as

$$
\bar{V}_{q}(0, z, s)=p \bar{P}_{q}^{(1)}(0, z, s) \bar{B}_{1}(s+\lambda-\lambda C(z)+\alpha) \bar{B}_{2}(s+\lambda-\lambda C(z)+\alpha)
$$

Using above equation, equation (53) becomes

$$
\bar{V}_{q}(z, s)=p \bar{P}_{q}^{(1)}(0, z, s) \bar{B}_{1}(s+\lambda-\lambda C(z)+\alpha) \bar{B}_{2}(s+\lambda-\lambda C(z)+\alpha)\left[\frac{1-\bar{V}(s+\lambda-\lambda C(z))}{(s+\lambda-\lambda C(z))}\right]
$$

Using equations (49) and (56) equation (41) becomes

$$
\bar{D}_{q}(z, s)=\alpha z \bar{P}_{q}^{(1)}(0, z, s)\left[\frac{1-\bar{B}_{1}(s+\lambda-\lambda C(z)+\alpha) \bar{B}_{2}(s+\lambda-\lambda C(z)+\alpha)}{(s+\lambda-\lambda C(z)+\alpha)(s+\lambda-\lambda C(z)+\eta)}\right]
$$

Using equation(49),equation(42) becomes

$$
\bar{R}_{q}(z, s)=\alpha z \eta \bar{P}_{q}^{(1)}(0, z, s)\left[\frac{1-\bar{B}_{1}(s+\lambda-\lambda C(z)+\alpha) \bar{B}_{2}(s+\lambda-\lambda C(z)+\alpha)}{(s+\lambda-\lambda C(z)+\alpha)(s+\lambda-\lambda C(z)+\beta)(s+\lambda-\lambda C(z)+\eta)}\right]
$$

Now using equations (56), (58) and (60) in equation (43) and solving for $\bar{P}_{q}^{(1)}(0, z, s)$

we get

$$
\bar{P}_{q}^{(1)}(0, z, s)=\frac{f_{1}(z, s) f_{2}(z, s) f_{3}(z, s)[(1-s \bar{Q}(s))+\lambda(C(z)-1) \bar{Q}(s)]}{D r}
$$

where $\mathrm{D}(\mathrm{z})=f_{1}(z, s) f_{2}(z, s) f_{3}(z, s)\{z-[(p z+q)(1-\theta)-\theta \bar{V}(s+\lambda-\lambda C(z))]$

$$
\left.\bar{B}_{1}\left[f_{1}(z, s)\right] \bar{B}_{2}\left[f_{1}(z, s)\right]\right\}-\alpha \beta \eta z\left(1-\bar{B}_{1}\left[f_{1}(z, s)\right] \bar{B}_{2}\left[f_{1}(z, s)\right]\right)
$$

$$
f_{1}(z, s)=s+\lambda-\lambda C(z)+\alpha
$$

$f_{2}(z, s)=s+\lambda-\lambda C(z)+\beta$

$f_{3}(z, s)=s+\lambda-\lambda C(z)+\eta$

Substituting the value of $\bar{P}_{q}^{(1)}(0, z, s)$ from equation (63) in to equation (47), (52), (60), (61)and (62) we get

$$
\begin{aligned}
& \bar{P}_{q}^{(1)}(z, s)=\frac{f_{2}(z, s) f_{3}(z, s)[(1-s \bar{Q}(s))+\lambda(C(z)-1) \bar{Q}(s)]\left[1-\bar{B}_{1}\left[f_{1}(z, s)\right]\right]}{D(z)} \\
& \bar{P}_{q}^{(2)}(z, s)=\frac{f_{2}(z, s) f_{3}(z, s)[(1-s \bar{Q}(s))+\lambda(C(z)-1) \bar{Q}(s)] \bar{B}_{1}\left[f_{1}(z, s)\right]\left[1-\bar{B}_{2}\left[f_{1}(z, s)\right]\right]}{D(z)} \\
& \bar{V}_{q}(z, s)=\frac{p f_{1}(z, s) f_{2}(z, s) f_{3}(z, s) \bar{B}_{1}\left[f_{1}(z, s)\right] \bar{B}_{2}\left[f_{1}(z, s)\right][(1-s \bar{Q}(s))+\lambda(C(z)-1) \bar{Q}(s)]\left[\frac{1-\bar{V}(s+\lambda-\lambda C(z))}{(s+\lambda-\lambda C(z))}\right]}{D(z)} \\
& \bar{D}_{q}(z, s)=f_{2}(z, s) \frac{\alpha z\left[1-\bar{B}_{1}\left[f_{1}(z, s)\right] \bar{B}_{2}\left[f_{1}(z, s)\right]\right][(1-s \bar{Q}(s))+\lambda(C(z)-1) \bar{Q}(s)]}{D(z)} \\
& \bar{R}_{q}(z, s)=\alpha \eta z \frac{\left[1-\bar{B}_{1}\left[f_{1}(z, s)\right] \bar{B}_{2}\left[f_{1}(z, s)\right]\right][(1-s \bar{Q}(s))+\lambda(C(z)-1) \bar{Q}(s)]}{D(z)}
\end{aligned}
$$

where $\mathrm{D}(\mathrm{z})$ is given by equation (64). 


\section{The Steady State Analysis}

In this section we shall derive the steady state probability distribution for our queueing model. To define the steady state probabilities, suppress the argument't' where ever it appears in the time dependent analysis.

By using well known Tauberian property

$$
L t_{s \rightarrow 0} s \bar{f}(s)=L t_{t \rightarrow \infty} f(t)
$$

multiplying both sides of equation (65),(66),(67),(68) and (69) by s and applying property (70) and simplifying, we get

$$
\begin{aligned}
& P_{q}^{(1)}(z)=\frac{f_{2}(z) f_{3}(z)[\lambda(C(z)-1)]\left[1-\bar{B}_{1}\left[f_{1}(z)\right]\right] Q}{D r} \\
& P_{q}^{(2)}(z)=\frac{f_{2}(z) f_{3}(z)[\lambda((C(z))-1)]\left[\bar{B}_{1}\left[f_{1}(z)\right]\right]\left[1-\bar{B}_{2}\left[f_{1}(z)\right]\right] Q}{D r} \\
& V_{q}(z)=p \frac{\left[f_{1}(z) f_{2}(z) f_{3}(z) \bar{B}_{1}\left[f_{1}(z)\right] \bar{B}_{2}\left[f_{1}(z)\right]\right][\bar{V}(\lambda-\lambda C(z))-1] Q}{D r} \\
& D_{q}(z)=\lambda \alpha z \frac{f_{2}(z)(C(z)-1)\left[1-\bar{B}_{1}\left[f_{1}(z)\right] \bar{B}_{2}\left[f_{1}(z)\right]\right] Q}{D r} \\
& R_{q}(z)=\frac{\lambda \alpha \eta z(C(z)-1)\left[1-\bar{B}_{1}\left[f_{1}(z)\right] \bar{B}_{2}\left[f_{1}(z)\right]\right] Q}{D r}
\end{aligned}
$$

where $\operatorname{Dr}=f_{1}(z) f_{2}(z) f_{3}(z)\left\{z-[(p z+q)(1-\theta)-\theta \bar{V}(\lambda-\lambda C(z))] \bar{B}_{1}\left[f_{1}(z)\right] \bar{B}_{2}\left[f_{1}(z)\right]\right\}$

$$
-\alpha \beta \eta z\left(1-\bar{B}_{1}\left[f_{1}(z)\right] \bar{B}_{2}\left[f_{1}(z)\right]\right)
$$

$f_{1}(z)=\lambda-\lambda C(z)+\alpha$

$f_{2}(z)=\lambda-\lambda C(z)+\beta$

$f_{3}(z)=\lambda-\lambda C(z)+\eta$

Let $W_{q}(z)$ denotes the probability generating function of queue size irrespective of the state of the system. Then adding(71),(72),(73),(74) and (75) we get

$$
\begin{aligned}
& W_{q}(z)=P_{q}^{(1)}(z)+P_{q}^{(2)}(z)+V_{q}(z)+D_{q}(z)+R_{q}(z) \\
& D r \frac{f_{2}(z) f_{3}(z)[\lambda(C(z)-1) Q]\left[1-\bar{B}_{1}\left[f_{1}(z)\right]\right]}{D r} \\
&+\frac{f_{2}(z) f_{3}(z)[\lambda((C(z))-1) Q]\left[\bar{B}_{1}\left[f_{1}(z)\right]\right]\left[1-\bar{B}_{2}\left[f_{1}(z)\right]\right]}{D r} \\
&+\frac{f_{2}(z) \lambda \alpha z(C(z)-1)\left[1-\bar{B}_{1}\left[f_{1}(z)\right] \bar{B}_{2}\left[f_{1}(z)\right]\right] Q}{D r} \\
&+ p \frac{\left[f_{1}(z) f_{2}(z) f_{3}(z) \bar{B}_{1}\left[f_{1}(z)\right] \bar{B}_{2}\left[f_{1}(z)\right]\right] Q[\bar{V}(\lambda-\lambda C(z))-1]}{D r} \\
&+\frac{\lambda \alpha \eta z(C(z)-1)\left[1-\bar{B}_{1}\left[f_{1}(z)\right] \bar{B}_{2}\left[f_{1}(z)\right]\right] Q}{D r}
\end{aligned}
$$

In order to obtain $\mathrm{Q}$, we use the normalization condition

$$
W_{q}(1)+Q=1
$$

We see that $\mathrm{z}=1, W_{q}(z)$ is indeterminate of the form $0 / 0$. We apply L'Hopital's rule in equation (78) where $\bar{B}_{i}(0)=1, i=1,2 ; \bar{V}(0)=1,-V^{\prime}(0)=E[V]$ the mean vacation time

Now

$$
P_{q}^{(1)}(1)=\frac{\lambda \beta \eta Q\left[1-\bar{B}_{1}(\alpha)\right] E(I)}{d r}
$$




$$
\begin{aligned}
& P_{q}^{(2)}(1)=\frac{\lambda \beta Q \eta\left[1-\bar{B}_{2}(\alpha)\right] \bar{B}_{1}(\alpha) E(I)}{d r} \\
& V_{q}(1)=p \frac{\lambda \alpha \beta \eta Q \bar{B}_{1}(\alpha) \bar{B}_{2}(\alpha) E(I) E(V)}{d r} \\
& D_{q}(1)=\frac{\lambda \alpha \beta Q E(I)\left(1-\bar{B}_{1}(\alpha) \bar{B}_{2}(\alpha)\right)}{d r} \\
& R_{q}(1)=\frac{\lambda \alpha \eta Q E(I)\left(1-\bar{B}_{1}(\alpha) \bar{B}_{2}(\alpha)\right)}{d r} \\
& W_{q}(1)=\frac{\lambda Q E(I)\left\{(\alpha \beta+\beta \eta+\eta \alpha)\left[1-\bar{B}_{1}(\alpha) \bar{B}_{2}(\alpha)\right]+\alpha \beta \eta p \bar{B}_{1}(\alpha) \bar{B}_{2}(\alpha) E(V)\right\}}{d r}
\end{aligned}
$$

where

$$
\begin{aligned}
& d r=(q+p \theta) \alpha \beta \eta \bar{B}_{1}(\alpha) \bar{B}_{2}(\alpha)-\lambda E(I)\left[(\alpha \beta+\beta \eta+\eta \alpha)\left[1-\bar{B}_{1}(\alpha) \bar{B}_{2}(\alpha)\right]\right] \\
& -\lambda \alpha \beta \eta E(I) E(V) \bar{B}_{1}(\alpha) \bar{B}_{2}(\alpha) \\
& Q=1-\frac{\lambda E(I)}{(q+p \theta)}\left[\frac{1}{\eta \bar{B}_{1}(\alpha) \bar{B}_{2}(\alpha)}+\frac{1}{\beta \bar{B}_{1}(\alpha) \bar{B}_{2}(\alpha)}+\frac{1}{\alpha \bar{B}_{1}(\alpha) \bar{B}_{2}(\alpha)}-\frac{1}{\eta}-\frac{1}{\beta}-\frac{1}{\alpha}+p E(V)\right]
\end{aligned}
$$

and the the utilization factor $\rho$ of the system is given by,

$$
\rho=\frac{\lambda E(I)}{(q+p \theta)}\left[\frac{1}{\eta \bar{B}_{1}(\alpha) \bar{B}_{2}(\alpha)}+\frac{1}{\beta \bar{B}_{1}(\alpha) \bar{B}_{2}(\alpha)}+\frac{1}{\alpha \bar{B}_{1}(\alpha) \bar{B}_{2}(\alpha)}-\frac{1}{\eta}-\frac{1}{\beta}-\frac{1}{\alpha}+p E(V)\right]
$$

where $\rho<1$ is the stability condition under which the steady state exists, equation (87) gives the probability that the server is idle. Substitute $\mathrm{Q}$ from equation (86) in equation (78), $W_{q}(z)$ have been completely and explicitly determined which is the the probability generating function of the queue size.

The average queue size

Let $L_{q}$ denote the mean number of customers in the queue under the steady state, then

$$
L_{q}=\left.\frac{d}{d z} W_{q}(z)\right|_{z=1}
$$

since this formula gives $0 / 0$ form, we write $W_{q}(z)=\frac{N(z)}{D(z)}$

where $\mathrm{N}(\mathrm{z})$ and $\mathrm{D}(\mathrm{z})$ are the numerator and denominator of the right hand side of equation (77) respectively, then we use

$$
L_{q}=\frac{D^{\prime}(1) N^{\prime \prime}(1)-N^{\prime}(1) D^{\prime \prime}(1)}{2\left[D^{\prime}(1)\right]^{2}}
$$

where primes and double primes in equation (88) denote first and second derivation at $\mathrm{z}=1$ respectively. Carrying out the derivatives at $\mathrm{z}=1$, we have

$$
\begin{aligned}
& N^{\prime}(1)=\lambda E(I) Q\left\{(\alpha \beta+\beta \eta+\eta \alpha)+\left(\bar{B}_{1}(\alpha) \bar{B}_{2}(\alpha)\right)[p \alpha \beta \eta E(V)-(\alpha \beta+\beta \eta+\eta \alpha)]\right\} \\
& N^{\prime \prime}(1)=2 Q[\lambda E(I)]^{2}\left\{\left(\frac{\alpha}{\lambda E(I)}-1\right)+\bar{B}_{1}(\alpha) \bar{B}_{2}(\alpha)\right. \\
& {\left[1-\frac{\alpha}{\lambda E(I)}-p \alpha \beta E(V)-p \beta \eta E(V)-p \eta \alpha E(V)+\frac{1}{2} p \alpha \beta \eta E\left(V^{2}\right)\right]} \\
& \left.+\left[\bar{B}_{2}(\alpha) \bar{B}_{1}^{\prime}(\alpha)+\bar{B}_{1}(\alpha) \bar{B}_{2}^{\prime}(\alpha)\right][(\alpha \beta+\beta \eta+\eta \alpha)-p \alpha \beta \eta E(V)]\right\} \\
& +\lambda Q E(I(I-1))\left\{(\alpha \beta+\beta \eta+\eta \alpha)+\bar{B}_{1}(\alpha) \bar{B}_{2}(\alpha)[p \alpha \beta \eta E(V)-(\alpha \beta+\beta \eta+\eta \alpha)]\right\} \\
& D^{\prime}(1)=-\lambda E(I)(\alpha \beta+\beta \eta+\eta \alpha)+\bar{B}_{1}(\alpha) \bar{B}_{2}(\alpha)\{(q+p \theta) \alpha \beta \eta \\
& +\lambda E(I)(\alpha \beta+\beta \eta+\eta \alpha)-p \alpha \beta \eta E(V)\}
\end{aligned}
$$




$$
\begin{aligned}
D^{\prime \prime}(1)= & 2[\lambda E(I)]^{2}\left\{\left(1-\frac{\alpha \beta+\beta \eta+\eta \alpha}{\lambda E(I)}\right)+\bar{B}_{1}(\alpha) \bar{B}_{2}(\alpha)\right. \\
& {\left[(-(q+p \theta)+p E(V))(\alpha \beta+\beta \eta+\eta \alpha)-\frac{1}{2} \alpha \beta \eta E\left(V^{2}\right)\right] } \\
& +\left[\bar{B}_{2}(\alpha) \bar{B}_{1}^{\prime}(\alpha)+\bar{B}_{1}(\alpha) \bar{B}_{2}^{\prime}(\alpha)\right]\left[-\frac{\alpha \beta \eta}{\lambda E(I)}-(q+p \theta)(\alpha \beta+\beta \eta+\eta \alpha)+\alpha \beta \eta p E(V)\right] \\
& +\lambda E(I(I-1))\left\{-(\alpha \beta+\beta \eta+\eta \alpha)+\bar{B}_{1}(\alpha) \bar{B}_{2}(\alpha)[(\alpha \beta+\beta \eta+\eta \alpha)-\alpha \beta \eta p E(V)]\right\}
\end{aligned}
$$

where $E\left(V^{2}\right)$ is the second moment of the vacation time and $\mathrm{Q}$ has been found in equation (86). Then if we substitute the values of $N^{\prime}(1), N^{\prime \prime}(1), D^{\prime}(1)$ and $D^{\prime \prime}(1)$ from equations(89),(90),(91) and (92) in to (88) equation we obtain $L_{q}$ in a closed form.

Mean waiting time of a customer could be found

$$
W_{q}=\frac{L_{q}}{\lambda}
$$

by using Little's formula.

\section{Conclusion}

We have proposed a single server with two stage heterogeneous service and Bernoulli scheduled vacation. There is a delay before the server get repaired which has been incorporated whenever the server meets break down. The probability generating function of transient solutions are obtained explicitly and along with this the steady state has also been analyzed. Further performance measures like average number of customers in the queue and the average waiting time of a customer in the queue are obtained.

\section{Acknowledgements.}

The authors are very much grateful to both the learned referees and the Managing Editor for their valuable the anonymous referees for their valuable comments to bring the paper in the present form.

\section{References}

[1] R.F. Anabosi and K.C. Madan, A single server queue with two types of service, Bernoulli schedule server vacations and a single vacation policy, Pakistan Journal of Statistics, 19(2003), 331 - 342.

[2] J.R. Artalejo and G. Choudhury, Steady state analysis of an M/G/1 queue with repeated attempts and two-phase service, Quality Technology and Quantitative Management, 1(2004), 189 - 199.

[3] Y. Baba, On the $M^{[X]} / G / 1$ queue with vacation time, Operations. Res. Lett., 5(1986), 93 - 98.

[4] A. BadamchiZadeh and G.H.A. Shahkar, Two Phases Queue System with Bernoulli Feedback and Bernoulli Schedule Server Vacation, Information and Management Sciences, 19(2008), 329-338.

[5] P.J. Burke, Delays in single-server queues with batch input, Operations.Res., 23(1975), 830 - 833

[6] Choi,Doo and Kim, Tae-Sung, Analysis of a Two- phase Queueing system with Vacation and Bernoulli feedback, Stochastic Analysis and Applications, 21(2003), 1009 - 1019.

[7] G. Choudhury and K. C. Madan, A two phase batch arrival queueing system with a vacation time under Bernoulli schedule, Applied Mathematics and Computation, 149(2004), 337 - 349.

[8] G. Choudhury and M. Paul, A two phase queueing system with Bernoulli feedback, Information and Management Science, 16(2005), $35-52$.

[9] G.Choudhury and K.C. Madan, A two-stage batch arrival queueing system with a modified Bernoulli schedule vacation under Npolicy, Mathematical and Computer Modelling, 42(2005), 71-85.

[10] G.Choudhury,L.Tadj and M.Paul, Steady state analysis of an $M^{[X]} / G / 1$ queue with two-phase service and Bernoulli vacation schedule under multiple vacation policy, Applied Mathematical Modelling, 31(2007), 1079-1091.

[11] B.T.Doshi, Queueing sysytem with vacation - A survey, Queueing System, 1(1986), 29-66.

[12] N. Igaki, Exponential two server queue with N policy and general vacation, Queueing Systems, 10(1992), 279 - 294.

[13] J. Keilson and L. D. Servi, Oscillating random walk models for GI/G/1 vacation systems with Bernoulli schedule, Journal of Applied Probability, 23(1986), 790 - 802.

[14] J. Keilson and L. D. Servi, Dynamic of the M/G/1 vacation model, Operation Research, 35(1987), July - August.

[15] B. Krishnakumar and D. Arivudainambi, An M/G/1/1 feedback queue with regular and optional services, Int. J. Inform. Manage. Sci., 12 (2001), $67-73$.

[16] V.G.Kulkarni and B.D.Choi, Retrial queues with server subject to breakdowns and repairs, Queueing Systems, 7(1990), 191-209.

[17] H. W.Lee, Bulk arrival queues with server vacations, Appl. Math. Modelling, 13(1989),374-377.

[18] H. Li and Y.Zhu, Analysis of M/G/1 queues with delayed vacations and exhaustive service discipline, European Journal of Operational Research, 92(1996), 125-134.

[19] K.C.Madan, A queueing system with random failures and delayed repairs, J. Ind. Statist. Assoc., 32(1994), 39-48.

[20] K. C.Madan, On a single server queue with two stage general heterogeneous service and deterministic server vacations, International Journal of System Sciences, 32(2001), 837-844. 
[21] K.C.Madan and R.F.Anabosi, A single server queue with two types of service, Bernoulli schedule server vacations and a single vacation policy, Pakistan Journal of Statistics, 19(2003), 331-342.

[22] F.A. Maraghi, K.C. Madan and K. Darby-Dowman, Batch arrival queueing system with random breakdowns and Bernoulli schedule server vacations having general vacation time distribution, International Journal of Information and Management Sciences, 20(2009), 55 - 70.

[23] P.R. Parthasarathy and R. Sudhesh, Transient solution of a multi server Poisson queue with N-policy, Computer and Mathematics with Applications, 55(2008), 550 - 562

[24] F.Rehab, K. C.Madan and A. L.Cormac, An M[X] /G/1 Queue with Bernoulli Schedule, General Vacation Times, Random Breakdowns, General Delay Times and General Repair Times, Applied mathematical Sciences, 6(2010), 35-51.

[25] D.D. Selvam and V. A. Sivasankaran, A two-phase queueing system with server vacations, Operations Research Letters, 15(1994), $163-168$.

[26] L. Takacs, A single server queue with feedback, The Bell system Technical Journal, 42(1963), 509 - 519.

[27] T. Takine and B. Sengupta, A single server queue with service interruptions, Queueing Systems, 26(1997), 285 - 300.

[28] H.Takagi, Time-dependent analysis of M/G/1 vacation models with exhaustive service, Queueing Systems,bf 6(1990), 369-390.

[29] A.M.K.Tarabia, Transient analysis of a non-empty M/M/1/N queue-An alternative approach, OPSEARCH, 38(2001), $431-438$.

[30] V. Thangaraj and S. Vanitha, M/G/1 Queue with Two-Stage Heterogeneous Service Compulsory Server Vacation and Random Breakdowns, Int. J. Contemp. Math. Sciences, 5(2010), 307-322.

[31] B.Vinck and H.Bruneel, System delay versus system content for discrete time queueing systems subject to server interruptions, European Journal of Operation Research, 175(2006), 362-375.

[32] K.H. Wang, T.Y. Wang and W.L. Pearn, Optimal control of the N-policy M/G/1 queueing system with server breakdowns and general startup times, Applied Mathematical Modelling, 31(2007), 2199 - 2212. 